\title{
Projective Synchronization Analysis of Drive-Response Coupled Dynamical Network with Multiple Time-Varying Delays via Impulsive Control
}

\author{
Song Zheng \\ School of Mathematics and Statistics, Zhejiang University of Finance and Economics, Hangzhou, Zhejiang 310018, China \\ Correspondence should be addressed to Song Zheng; zhengs02012@gmail.com
}

Received 17 February 2014; Revised 4 April 2014; Accepted 16 April 2014; Published 15 May 2014

Academic Editor: Derui Ding

Copyright (C) 2014 Song Zheng. This is an open access article distributed under the Creative Commons Attribution License, which permits unrestricted use, distribution, and reproduction in any medium, provided the original work is properly cited.

\begin{abstract}
The problem of projective synchronization of drive-response coupled dynamical network with delayed system nodes and multiple coupling time-varying delays is investigated. Some sufficient conditions are derived to ensure projective synchronization of driveresponse coupled network under the impulsive controller by utilizing the stability analysis of the impulsive functional differential equation and comparison theory. Numerical simulations on coupled time delay Lorenz chaotic systems are exploited finally to illustrate the effectiveness of the obtained results.
\end{abstract}

\section{Introduction}

In the past few years, it is found that synchronization is one of the most important and interesting collective behaviors of complex networks and has been extensively investigated in different fields of engineering and sociology [111]. Meanwhile, many kinds of synchronization have been proposed, such as complete synchronization, phase synchronization, lag synchronization, anticipated synchronization, cluster synchronization, generalized synchronization, and projective synchronization. And various control methods such as adaptive control [12-15], impulsive control [1620], pinning control [21-25], and intermittent control [26, 27] have been reported to achieve the different kinds of synchronization for complex networks.

In projective synchronization, the drive-response systems can be synchronized up to a scaling factor. Due to the potential applications in secure communication, the projective synchronization has been extremely investigated including chaotic systems [28-32] and complex dynamical networks [33-40]. Xu [29] studied the projective synchronization in coupled partially linear systems via adaptive feedback control. Furthermore, $\mathrm{Hu}$ et al. [35] introduced a driveresponse dynamical network model and investigated its projective synchronization properties using pinning control to obtain the desired scaling factor. A short time later, they investigated projective cluster synchronization in a driveresponse dynamical network model with coupled partially linear chaotic systems [36]. The impulsive projective synchronization between the drive system and response dynamical network without the time delay was investigated in [37]. It is noted that in practical cases time delays are often encountered. Ignoring them may lead to design flaws and incorrect analysis conclusions. Consequently, time delay case should be considered. Recently, Sun et al. [38] studied the projective synchronization in drive-response dynamical networks of partially linear systems with time-varying coupling delay. Chen et al. [39] proposed projective (anticipatory, exact, and lag) synchronization criteria for a drive-response complex network with different scale factors. Moreover, in much of the literature, time delays in the couplings are considered; however, time delays in the dynamical nodes $[32,40]$, which are more complex, are still relatively unexplored. Zheng [40] investigated the adaptive-impulsive projective synchronization of drive-response delayed complex dynamical networks with time-varying coupling. Cao et al. [32] proposed projective synchronization of a class of delayed chaotic systems via impulsive control, where the drive-response system can be synchronized to within a scaling factor. On the other hand, it is well known that the impulsive control method $[16-20,32$, 
37, 40] is discontinuous, effective, robust, and low-cost and has been widely applied in many fields, such as information science, control systems, communication security, and space techniques. To the best of the authors' knowledge, projective synchronization of a drive-response coupled dynamical network model with time-delayed dynamical nodes and delayed coupling has not been reported via impulsive control. Moreover, in many networks, nodes may influence each other not only by nondelayed state information but also by delayed state information. Therefore, both nondelayed coupling and multiple delayed couplings should be also considered.

Motivated by the above discussions, this paper aims to handle the problem of the impulsive projective synchronization for a drive-response coupled dynamical network with dynamical nodes delay and both nondelayed coupling and multiple delayed couplings. The sufficient conditions for projective synchronization are derived analytically by using the stability analysis of the impulsive functional differential equation, and an impulsive controller is designed. Analytical results show that drive-response coupled dynamical networks with multiple time delays can realize projective synchronization within a scaling factor.

Notations. Throughout this paper, $R^{n}$ and $R^{n \times n}$ denote, respectively, the $n$-dimensional Euclidean space and the set of all $n \times n$ real matrices. The superscript $T$ denotes matrix or vector transposition. $I_{n}$ is the $n \times n$ identity matrix. $\lambda_{\max }(A)$ means the maximum eigenvalue of matrix $A$. The Euclidean norm in $R^{n}$ is defined as $\|\cdot\|$; for vector $x \in R^{n},\|x\|=x^{T} x$ and for matrix $A \in R^{n \times n},\|A\|=\sqrt{\lambda_{\max }\left(A^{T} A\right)} \cdot \mu(A)=$ $\lambda_{\text {max }}\left(A^{T}+A\right)$, and the symbol $\otimes$ is the Kronecker product of two matrices. The upper bound is denoted by sup. The matrices, if their dimensions are not explicitly stated, are assumed to have appropriate dimensions.

The rest of this paper is organized as follows: in Section 2, the model of drive-response coupled dynamical network with time-varying delays is introduced and some necessary preliminaries are given. In Section 3, the projective synchronization criteria are derived by using impulsive control. Numerical simulations are shown in Section 4. The conclusion is finally drawn in Section 5.

\section{Model Description and Preliminaries}

The projective synchronization in coupled partially linear delayed chaotic systems via impulsive control is studied in [32]. Inspired by [32], the drive-response coupled network model with dynamical nodes delay and multiple coupling delays, in which dynamical nodes are partially linear timedelayed chaotic systems, is described as follows:

$$
\begin{aligned}
\dot{u}^{d}= & M(z) \cdot u^{d}(t)+\gamma \Gamma\left(u^{d}(t-\tau(t))-u^{d}(t)\right), \\
\dot{z}(t)= & f\left(u^{d}(t), u^{d}(t-\tau(t)), z(t), z(t-\tau(t))\right), \\
\dot{u}_{i}^{r}= & M(z) \cdot u_{i}^{r}(t)+\gamma \Gamma\left(u_{i}^{r}(t-\tau(t))-u_{i}^{r}(t)\right) \\
& +c \sum_{j=1}^{N} c_{i j} A u_{j}^{r}(t)
\end{aligned}
$$

$$
\begin{aligned}
& +c^{\tau_{1}} \sum_{j=1}^{N} c_{i j}^{\tau_{1}} A^{\tau_{1}} u_{j}^{r}\left(t-\tau_{1}(t)\right)+\cdots \\
& +c^{\tau_{m}} \sum_{j=1}^{N} c_{i j}^{\tau_{m}} A^{\tau_{m}} u_{j}^{r}\left(t-\tau_{m}(t)\right),
\end{aligned}
$$

where the drive system and the response network systems are linked through the variable $z(t) \in R^{1} \cdot u^{d}(t) \in R^{n}$ is the state variables of the drive system, and $u_{i}^{r}(t) \in R^{n}$ denotes the state variables of the $i$ th node in the response network systems. The $d$ and $r$ stand for the drive system and response system, respectively, and $M(z) \in R^{n \times n}$ is a matrix which depends on the variable $z(t)$. The constant $\gamma$ is a positive constant and $\Gamma \in R^{n \times n}$ a matrix. The constants $c>0$ and $c^{\tau_{l}}>0(l=$ $1,2, \ldots, m)$ are the nondelayed and the delayed coupling strength to be adjusted, respectively, and the time-varying delays $\tau(t)$ and $\tau^{l}(t)$ are bounded by a known constant; that is, $0 \leq \tau(t) \leq \tau, 0 \leq \tau_{l}(t) \leq \tau_{l}$. $A \in R^{n \times n}$ and $A^{\tau_{l}} \in$ $R^{n \times n}$ represent the nondelayed and delayed inner-coupling matrices, respectively. $C=\left(c_{i j}\right)_{N \times N}$ and $C^{\tau_{l}}=\left(c_{i j}^{\tau_{l}}\right)_{N \times N}$ are the nondelayed and delayed outer-coupling configuration matrices, respectively, in which $c_{i j} \neq 0\left(c_{i j}^{\tau_{l}} \neq 0\right)$ if there is a link from node $i$ to node $j(i \neq j)$ and $c_{i j}(t)=0\left(c_{i j}^{\tau_{l}}=0\right)$ otherwise; the diagonal elements of matrix $C\left(C^{\tau_{l}}\right)$ are given by $c_{i i}=-\sum_{j=1, j \neq i}^{N} c_{i j},\left(c_{i i}^{\tau_{l}}=-\sum_{j=1, j \neq i}^{N} c_{i j}^{\tau_{l}}\right), i=1,2, \ldots, N$, $l=1,2, \ldots, m$.

Remark 1. In this paper, it should be pointed out that we do not require that the time-varying delay is a differential function with a bound of its derivative, which means that the timevarying delays include a wide range of functions. Moreover, the coupling configuration matrices are not assumed to be symmetric or irreducible.

In order to derive our main results, some necessary definitions and lemmas are needed.

Definition 2. The projective synchronization is said to take place in drive-response coupled network (1), if there exists constant $\alpha(\alpha \neq 0)$ such that $\lim _{t \rightarrow \infty}=\left\|u_{i}^{r}(t)-\alpha u^{d}(t)\right\|=0$ for all $i$, where $\alpha$ is the scaling factor.

Lemma 3 (see [41]). Let $0 \leq \tau(t), \tau_{1}(t), \tau_{2}(t), \ldots, \tau_{m}(t) \leq$ $\tilde{\tau}, \tilde{\tau}=\max \left(\tau, \tau_{1}, \tau_{2}, \ldots, \tau_{m}\right), F\left(t, u, \bar{u}_{1}, \ldots, \bar{u}_{m}\right): R^{+} \times$ $\overbrace{R \times \cdots}^{m+1}$

$\overbrace{R \times \cdots \times R} \rightarrow \quad R$ be nondecreasing in $\bar{u}_{i}$ for each fixed $\left(t, u, \bar{u}_{1}, \ldots, \bar{u}_{i-1}, \bar{u}_{i+1}, \bar{u}_{m}\right), i=1,2, \ldots, m$, and let $I_{k}(u):$ $R \rightarrow R$ be nondecreasing in $u$. Suppose that $u(t)$ and $v(t)$ satisfy

$$
\begin{gathered}
D^{+} u(t) \leq F\left(t, u(t), u_{1}\left(t-\tau_{1}(t)\right), \ldots, u_{m}\left(t-\tau_{m}(t)\right)\right), \\
t \geq 0, \\
u\left(t_{k}\right) \leq I_{k} u\left(t_{k}\right), \quad k \in N, \\
D^{+} v(t)>F\left(t, v(t), v_{1}\left(t-\tau_{1}(t)\right), \ldots, v_{m}\left(t-\tau_{m}(t)\right)\right), \\
v\left(t_{k}^{+}\right) \geq I_{k} v\left(t_{k}\right), \quad k \in N,
\end{gathered}
$$


where the right and upper Dini's derivative $D^{+} u(t)$ is defined as $D^{+} u(t)=\varlimsup_{\lim _{h \rightarrow 0^{+}}}((u(t+h)-u(t)) / h)$, where $h \rightarrow 0^{+}$ means that $h$ approaches zero from the right-hand side. Then $u(t) \leq v(t)$ for $-\tau \leq t \leq 0$ implies that $u(t) \leq v(t)$ for $t \geq 0$.

\section{Projective Synchronization Analysis}

This section addresses the implementation of projective synchronization between the drive and response networks with time delay characteristics. By taking a theoretical approach based on the classic Lyapunov stability theory, we derive the criteria of network projective synchronization and present an impulsive control scheme.

By selecting proper control gain matrix $B_{k_{i}} \in R^{n \times n}$, the drive-response network (1) can be rewritten as the following controlled impulsive differential equation:

$$
\begin{aligned}
\dot{u}^{d}(t)= & M(z) \cdot u^{d}(t)+\gamma \Gamma\left(u^{d}(t-\tau(t))-u^{d}(t)\right), \\
\dot{z}(t)= & f\left(u^{d}(t), u^{d}(t-\tau(t)), z(t), z(t-\tau(t))\right), \\
\dot{u}_{i}^{r}= & M(z) \cdot u_{i}^{r}(t)+\gamma \Gamma\left(u_{i}^{r}(t-\tau(t))-u_{i}^{r}(t)\right) \\
& +c \sum_{j=1}^{N} c_{i j} A u_{j}^{r}(t) \\
& +\sum_{l=1}^{m} \sum_{j=1}^{N} c^{\tau_{l}} c_{i j}^{\tau_{l}} A^{\tau_{l}} u_{j}^{r}\left(t-\tau_{l}(t)\right), \quad t \neq t_{k}, \\
\Delta e_{i} & =B_{i_{k}} e_{i}\left(t_{k}^{-}\right), \quad t=t_{k}, k=1,2, \ldots,
\end{aligned}
$$

where the impulsive time instants $t_{k}$ satisfy $t_{0}<t_{1}<t_{2}<$ $\cdots<t_{k}<\cdots$, and $\lim _{k \rightarrow \infty} t_{k}=+\infty, \Delta u_{i}^{r}=u_{i}^{r}\left(t_{k}^{+}\right)-$ $u_{i}^{r}\left(t_{k}^{-}\right)$is the control law in which $u_{i}^{r}\left(t_{k}^{+}\right)=\lim _{t \rightarrow t_{k}^{+}} u_{i}^{r}(t)$ and $u_{i}^{r}\left(t_{k}^{-}\right)=\lim _{t \rightarrow t_{k}^{-}} u_{i}^{r}(t)$. Without loss of generality, we assume that $\lim _{t \rightarrow t_{k}^{-}} u_{i}^{r}(t)=u_{i}^{r}\left(t_{k}\right)$, which means that the solution of (3) is left continuous at time $t_{k}$.

Remark 4. Compared with continuous control, discontinuous control, including impulsive control and intermittent control, is effective, practical, and applicable in many areas, especially for secure communication. Impulsive controller has a relatively simple structure and is easy to implement. In an impulsive synchronization scheme, the response system receives the information from the drive system only in discrete times and the amount of conveyed information is, therefore, decreased. This is very advantageous in practice due to reduced control cost.

Letting projective synchronization error be $e_{i}(t)=u_{i}^{r}(t)-$ $\alpha u^{d}(t)$, the error dynamical network is characterized by

$$
\begin{aligned}
\dot{e}_{i}(t)= & M(z) e_{i}(t)+\gamma \Gamma\left(e_{i}\left(t-\tau(t)-e_{i}(t)\right)\right) \\
& +c \sum_{j=1}^{N} c_{i j} A e_{j}(t) \\
& +\sum_{l=1}^{m} \sum_{j=1}^{N} c^{\tau_{l}} C_{i j}^{\tau_{l}} e_{j}\left(t-\tau_{l}(t)\right), \quad t \neq t_{k},
\end{aligned}
$$

$$
\begin{gathered}
\dot{z}(t)=f\left(u^{d}(t), u^{d}(t-\tau(t)), z(t), z(t-\tau(t))\right), \\
\Delta e_{i}=B_{i_{k}} e_{i}\left(t_{k}\right), \quad t=t_{k}, k=1,2, \ldots
\end{gathered}
$$

Let $e(t)=\left(e_{1}^{T}(t), e_{2}^{T}(t), \ldots, e_{N}^{T}(t)\right)^{T}$, and then (4) can be rewritten in the Kronecker product form as

$$
\begin{gathered}
\dot{e}(t)=\left[\left(I_{N} \otimes(M(z)-\gamma \Gamma)\right)+c(C \otimes A)\right] e(t) \\
+\left(I_{N} \otimes \gamma \Gamma\right) e(t-\tau(t)) \\
+\sum_{l=1}^{m} c^{\tau_{l}}\left(C^{\tau_{l}} \otimes A^{\tau_{l}}\right) e\left(t-\tau_{l}(t)\right), \quad t \neq t_{k}, \\
\dot{z}(t)=f\left(u^{d}(t), u^{d}(t-\tau(t)), z(t), z(t-\tau(t))\right), \\
e\left(t_{k}^{+}\right)=\left(I_{N n}+\left(I_{N} \otimes B_{i_{k}}\right)\right) e\left(t_{k}\right), \quad t=t_{k}, \quad k=1,2, \ldots
\end{gathered}
$$

Let $C\left(\left[t_{0}-\tilde{\tau}, t_{0}\right], R^{n}\right)$ be the Banach space of continuous vector-valued functions mapping the interval $\left[t_{0}-\tilde{\tau}, t_{0}\right]$ into $R^{n}$ with a topology of uniform convergence. $\|\phi\|=$ $\sup _{t_{0}-\tilde{\tau} \leq s \leq t_{0}}\|\phi(s)\|$ is used to denote the norm of a function $\phi \in C\left(\left[t_{0}-\tilde{\tau}, t_{0}\right], R^{n}\right)$. For functional differential equation (5), its initial condition is given by $e(s)=\phi(s) \in C\left(\left[t_{0}-\tilde{\tau}, t_{0}\right], R^{n}\right)$. For simplicity, it is assumed that $e(t)$ is continuous at $t_{0}=0$.

Letting $0<\rho=\sup \left\{t_{k}-t_{k-1}\right\}<\infty, \lambda_{\max }\left[\left(I_{N n}+\left(I_{N} \otimes\right.\right.\right.$ $\left.\left.\left.B_{k_{i}}\right)\right)^{T}\left(I_{N n}+\left(I_{N} \otimes B_{k_{i}}\right)\right)\right] \leq \eta$, based on the theory of impulsive functional differential equation and comparison method, we have the following results.

Theorem 5. For given synchronization scaling factor $\alpha$, projective synchronization in the drive-response coupled dynamical network with multiple time-varying delays model will occur if the following inequalities hold:

$$
\begin{gathered}
0<\eta<1, \\
\frac{\ln \eta}{\rho}+\sup \left[\mu(M(z))+\frac{2}{\sqrt{\eta}}\left\|I_{N} \otimes \gamma \Gamma\right\|\right. \\
\left.+\sum_{l=1}^{m} \frac{2}{\sqrt{\eta}}\left\|c^{\tau_{l}}\left(C^{\tau_{l}} \otimes A^{\tau_{l}}\right)\right\|\right]<0,
\end{gathered}
$$

where $M(z)=\left(I_{N} \otimes(M(z)-\gamma \Gamma)\right)+c(C \otimes A)$, and then the error system (5) can converge globally exponentially to a decay rate $\lambda / 2$, where $\lambda>0$ is the solution of $\lambda-a+(1 / \sqrt{\eta})\left\|I_{N} \otimes \gamma \Gamma\right\| e^{\lambda \tau}+$ $\sum_{l=1}^{m}(1 / \sqrt{\eta})\left\|c^{\tau_{l}}\left(C^{\tau_{l}} \otimes A^{\tau_{l}}\right)\right\| e^{\lambda \tau_{l}}=0$ with $a=-\sup [\mu(M(z))+$ $\left.(2 / \sqrt{\eta})\left\|I_{N} \otimes \gamma \Gamma\right\|+\sum_{l=1}^{m}(2 / \sqrt{\eta})\left\|c^{\tau_{l}}\left(C^{\tau_{l}} \otimes A^{\tau_{l}}\right)\right\|\right]-(\ln \eta / \rho)$. That is to say, the coupled dynamical drive-response network with time-varying delays can realize the projective synchronization via impulsive control.

Proof. Consider the following Lyapunov candidate function:

$$
V(t)=e^{T}(t) e(t)
$$


For $t \in\left[t_{k-1}, t_{k}\right), k \in N$, differentiating $V(t)$ along the solution of (5), one obtains that

$$
\begin{aligned}
\dot{V}(t)= & 2 e^{T}(t) \dot{e}(t) \\
= & 2 e^{T}(t)\left[\left(I_{N} \otimes(M(z)-\gamma \Gamma)\right)+c(C \otimes A)\right] e(t) \\
& +2 e^{T}(t)\left(I_{N} \otimes \gamma \Gamma\right) e(t-\tau(t)) \\
& +2 \sum_{l=1}^{m} e^{T}(t) c^{\tau_{l}}\left(C^{\tau_{l}} \otimes A^{\tau_{l}}\right) e\left(t-\tau_{l}(t)\right) .
\end{aligned}
$$

It is clear that

$$
\begin{aligned}
& 2 e^{T}(t)\left(I_{N} \otimes \gamma \Gamma\right) e(t-\tau(t)) \\
& \leq \varsigma e^{T}(t) e(t) \\
& \quad+\frac{1}{\varsigma} e^{T}(t-\tau(t))\left(I_{N} \otimes \gamma \Gamma\right)^{T}\left(I_{N} \otimes \gamma \Gamma\right) e(t-\tau(t)) \\
& 2 \sum_{l=1}^{m} e^{T}(t) c^{\tau_{l}}\left(C^{\tau_{l}} \otimes A^{\tau_{l}}\right) e\left(t-\tau_{l}(t)\right) \\
& \leq \sum_{l=1}^{m} \varsigma_{l} e^{T}(t) e(t) \\
& \quad+\sum_{l=1}^{m} \frac{1}{\varsigma_{l}} e^{T}\left(t-\tau_{l}(t)\right)\left(c^{\tau_{l}}\left(C^{\tau_{l}} \otimes A^{\tau_{l}}\right)\right)^{T} \\
& \times\left(c^{\tau_{l}}\left(C^{\tau_{l}} \otimes A^{\tau_{l}}\right)\right) e\left(t-\tau_{l}(t)\right) .
\end{aligned}
$$

From the definition of $M(z)$, then, one obtains

$$
\begin{aligned}
\dot{V}(t) \leq & e^{T}(t)\left[M(z)^{T}+M(z)\right] e(t)+\varsigma e^{T}(t) e(t) \\
& +\sum_{l=1}^{m} \varsigma_{l} e^{T}(t) e(t) \\
& +\frac{1}{\varsigma} e^{T}(t-\tau(t))\left(I_{N} \otimes \gamma \Gamma\right)^{T}\left(I_{N} \otimes \gamma \Gamma\right) e(t-\tau(t)) \\
& +\sum_{l=1}^{m} \frac{1}{\varsigma_{l}} e^{T}\left(t-\tau_{l}(t)\right)\left(c^{\tau_{l}}\left(C^{\tau_{l}} \otimes A^{\tau_{l}}\right)\right)^{T} \\
\leq & \quad \sup \left[\mu\left(c^{\tau_{l}}\left(C^{\tau_{l}} \otimes A^{\tau_{l}}\right)\right) e\left(t-\tau_{l}(t)\right)\right. \\
& \left.+\frac{1}{\varsigma}\left\|I_{N} \otimes \gamma \Gamma\right\|^{2} V(t-\tau(t))+\varsigma+\sum_{l=1}^{m} \varsigma_{l}\right] V(t) \\
& +\sum_{l=1}^{m} \frac{1}{\varsigma_{l}}\left\|c^{\tau_{l}}\left(C^{\tau_{l}} \otimes A^{\tau_{l}}\right)\right\|^{2} V\left(t-\tau_{l}(t)\right) .
\end{aligned}
$$

When $t=t_{k}, k \in N$, one has

$$
\begin{aligned}
V\left(t_{k}^{+}\right) & =e^{T}\left(t_{k}\right)\left(I_{N n}+\left(I_{N} \otimes B_{k}\right)\right)^{T}\left(I_{N n}+\left(I_{N} \otimes B_{k}\right)\right) e\left(t_{k}\right) \\
& \leq \eta e^{T}\left(t_{k}\right) e\left(t_{k}\right) \\
& =\eta V\left(t_{k}\right), \quad k=1,2, \ldots .
\end{aligned}
$$

For any $\varepsilon>0$, let $v(t)$ be a unique solution of the following impulsive delay system:

$$
\begin{gathered}
\dot{v}(t)=\sup \left[\mu(M(z))+\varsigma+\sum_{l=1}^{m} \varsigma_{l}\right] v(t) \\
+\frac{1}{\varsigma}\left\|I_{N} \otimes \gamma \Gamma\right\|^{2} v(t-\tau(t)) \\
+\sum_{l=1}^{m} \frac{1}{\varsigma_{l}}\left\|c^{\tau_{l}}\left(C^{\tau_{l}} \otimes A^{\tau_{l}}\right)\right\|^{2} v\left(t-\tau_{l}(t)\right)+\varepsilon, \quad t \neq t_{k}, \\
v\left(t_{k}^{+}\right)=\eta v\left(t_{k}\right), \quad k \in N, \\
v(s)=\|\phi(s)\|^{2}, \quad-\tilde{\tau} \leq s \leq 0,
\end{gathered}
$$

where $\phi(s)=\left(\phi_{1}^{T}(s), \phi_{2}^{T}(s), \ldots, \phi_{N}^{T}(s)\right)^{T}$.

Since $V(s) \leq\|\phi(s)\|^{2}$ for $-\widetilde{\tau} \leq s \leq 0$, it follows from (12)(13) and Lemma 3 that

$$
0 \leq V(t) \leq v(t), \quad \text { for } t \geq 0
$$

By the formula for the variation of parameters, one obtains $v(t)$ from (13) that

$$
\begin{aligned}
& v(t) \\
& =\omega(t, 0) v(0) \\
& +\int_{0}^{t} \omega(t, s)\left(\frac{1}{\varsigma}\left\|I_{N} \otimes \gamma \Gamma\right\|^{2} v(s-\tau(s))\right. \\
& \left.\quad+\sum_{l=1}^{m} \frac{1}{\varsigma_{l}}\left\|c^{\tau_{l}}\left(C^{\tau_{l}} \otimes A^{\tau_{l}}\right)\right\|^{2} v\left(t-\tau_{l}(s)\right)+\varepsilon\right) d s,
\end{aligned}
$$

where $\omega(t, s), 0 \leq s \leq t$, is Cauchy matrix of the linear system

$$
\begin{gathered}
\dot{\varsigma}(t)=\sup \left[\mu(M(z))+\varsigma+\sum_{l=1}^{m} \varsigma_{l}\right] \varsigma(t), \quad t \neq t_{k}, \\
\varsigma\left(t_{k}^{+}\right)=\eta \varsigma\left(t_{k}\right), \quad k \in N .
\end{gathered}
$$


According to the representation of the Cauchy matrix, we get the following estimation of $\omega(t, s)$, since $0<\eta<1$ and $t_{k}-t_{k-1} \leq \rho$,

$$
\begin{aligned}
\omega(t, s) & =e^{\sup \left[\mu(M(z))+\varsigma+\sum_{l=1}^{m} \varsigma_{l}\right](t-s)} \prod_{s<t_{k} \leq t} \eta \\
& \leq e^{(-a-(\ln \eta / \rho))(t-s)} \eta^{(((t-s) / \rho)-1)} \\
& =\frac{1}{\eta} e^{-a(t-s)}, \quad 0 \leq s \leq t,
\end{aligned}
$$

For simplicity, let $\gamma=(1 / \eta) \sup _{-\tilde{\tau} \leq s \leq 0}\left\{\|\phi(s)\|^{2}\right\}, \varsigma=$ $(1 / \sqrt{\eta})\left\|I_{N} \otimes \gamma \Gamma\right\|, \varsigma_{l}=(1 / \sqrt{\eta})\left\|c^{\tau_{l}}\left(C^{\tau_{l}} \otimes A^{\tau_{l}}\right)\right\|$, from (15) and (17), one has

$$
\begin{aligned}
& v(t) \leq \frac{1}{\eta} e^{-a t} v(0) \\
& +\int_{0}^{t} e^{-a(t-s)} \frac{1}{\eta} \\
& \times\left[\frac{1}{\varsigma}\left\|I_{N} \otimes \gamma \Gamma\right\|^{2} v(s-\tau(s))\right. \\
& \left.+\sum_{l=1}^{m} \frac{1}{\varsigma_{l}}\left\|c^{\tau_{l}}\left(C^{\tau_{l}} \otimes A^{\tau_{l}}\right)\right\|^{2} v\left(t-\tau_{l}(s)\right)+\varepsilon\right] d s \\
& \leq \int_{0}^{t} e^{-a(t-s)}\left[\begin{array}{c}
\varsigma v(s-\tau(s)) \\
\left.+\sum_{l=1}^{m} \varsigma_{l} v\left(s-\tau_{l}(s)\right)+\frac{\varepsilon}{\eta}\right] d s .
\end{array}\right.
\end{aligned}
$$

Defining $\varphi(\lambda)=\lambda-a+\varsigma e^{\lambda \tau}+\sum_{l=1}^{m} \varsigma_{l} e^{\lambda \tau}$, from (7), one has $a, \varsigma, \sum_{l=1}^{m} \varsigma_{l}$ and $a-\varsigma-\sum_{l=1}^{m} \varsigma_{l}>0$, and also $\varphi(0)<0$, $\varphi(+\infty)>0, \varphi^{\prime}(\lambda)=1+\varsigma \tau e^{\lambda \tau}+\sum_{l=1}^{m} \varsigma_{l} \tau_{l} e^{\lambda \tau_{l}}>0$. Therefore, there exists a unique solution $\lambda>0$ such that $\varphi(\lambda)=0$.

On the other hand, since $\varepsilon>0, \lambda>0, a-\varsigma-\sum_{l=1}^{m} \varsigma_{l}>0$, and $(1 / \eta)>1$, one has

$$
\begin{aligned}
v(t) & \leq \frac{1}{\eta} \sup _{-\tilde{\tau} \leq s \leq 0} v(s) \\
& <\gamma e^{-\lambda t}+\frac{\varepsilon}{\eta\left(a-\varsigma-\sum_{l=1}^{m} \varsigma_{l}\right)}, \quad-\widetilde{\tau} \leq t \leq 0 .
\end{aligned}
$$

In the following, we will prove that the following inequality holds:

$$
v(t)<\gamma e^{-\lambda t}+\frac{\varepsilon}{\eta\left(a-\varsigma-\sum_{l=1}^{m} \varsigma_{l}\right)}, \quad t>0 .
$$

If (20) is not true; that is, it is assumed that there exists a $t^{*}>0$ such that

$$
\begin{gathered}
v\left(t^{*}\right) \geq \gamma e^{-\lambda t^{*}}+\frac{\varepsilon}{\eta\left(a-\varsigma-\sum_{l=1}^{m} \varsigma_{l}\right)}, \\
v(t)<\gamma e^{-\lambda t}+\frac{\varepsilon}{\eta\left(a-\varsigma-\sum_{l=1}^{m} \varsigma_{l}\right)}, \quad t<t^{*} .
\end{gathered}
$$

From (18) and (22), one has

$$
\begin{aligned}
& v\left(t^{*}\right) \\
& \leq \gamma e^{-a t^{*}} \\
& +\int_{0}^{t^{*}} e^{-a\left(t^{*}-s\right)}\left[\varsigma v(s-\tau(s))+\sum_{l=1}^{m} \varsigma_{l} v\left(s-\tau_{l}(s)\right)+\frac{\varepsilon}{\eta}\right] d s \\
& <e^{-a t^{*}}\left(\gamma+\frac{\varepsilon}{\eta\left(a-\varsigma-\sum_{l=1}^{m} \varsigma_{l}\right)}\right) \\
& +e^{-a t^{*}} \int_{0}^{t^{*}} e^{a s}\left[\varsigma\left(\gamma e^{-\lambda(s-\tau(s))}+\frac{\varepsilon}{\eta\left(a-\varsigma-\sum_{l=1}^{m} \varsigma_{l}\right)}\right)\right. \\
& +\sum_{l=1}^{m} \varsigma_{l}\left(\gamma e^{-\lambda\left(s-\tau_{l}(s)\right)}+\frac{\varepsilon}{\eta\left(a-\varsigma-\sum_{l=1}^{m} \varsigma_{l}\right)}\right) \\
& \left.+\frac{\varepsilon}{\eta}\right] d s \\
& \leq e^{-a t^{*}}\left(\gamma+\frac{\varepsilon}{\eta\left(a-\varsigma-\sum_{l=1}^{m} \varsigma_{l}\right)}\right) \\
& +e^{-a t^{*}} \int_{0}^{t^{*}} e^{a s}\left[\varsigma e^{-\lambda(s-\tau(s))}+\sum_{l=1}^{m} \varsigma_{l} e^{-\lambda\left(s-\tau_{l}(s)\right)}\right. \\
& \left.+\frac{\left(\varsigma+\sum_{l=1}^{m} \varsigma_{l}\right) \varepsilon}{\eta\left(a-\varsigma-\sum_{l=1}^{m} \varsigma_{l}\right)}+\frac{\varepsilon}{\eta}\right] d s \\
& \leq e^{-a t^{*}}\left\{\gamma+\frac{\varepsilon}{\eta\left(a-\varsigma-\sum_{l=1}^{m} \varsigma_{l}\right)}\right. \\
& +\gamma\left(\varsigma e^{\lambda \tau}+\sum_{l=1}^{m} \varsigma_{l} e^{\lambda \tau_{l}}\right) \int_{0}^{t^{*}} e^{(a-\lambda) s} d s \\
& \left.+\frac{a \varepsilon}{\eta\left(a-\varsigma-\sum_{l=1}^{m} \varsigma_{l}\right)} \int_{0}^{t^{*}} e^{a s} d s\right\} \\
& <\gamma e^{-\lambda t^{*}}+\frac{\varepsilon}{\eta\left(a-\varsigma-\sum_{l=1}^{m} \varsigma_{l}\right)},
\end{aligned}
$$

which contradicts (21), so (20) holds. Letting $\varepsilon \rightarrow 0$, we get

$$
V(t)=\|e(t)\|^{2} \leq v(t) \leq \gamma e^{-\lambda t} .
$$

Therefore, we have

$$
\|e(t)\| \leq \sqrt{\gamma} e^{-(\lambda / 2) t}, \quad t \geq 0 .
$$

When $t \rightarrow \infty$, the error system (5) is global exponential asymptotically stable, which implies that the drive-response coupled networks (1) achieve projective synchronization with a scaling factor via impulsive control. This completes the proof of Theorem 5 .

From Theorem 5, it is easy to obtain the following corollaries for the drive-response coupled dynamical network without the nodes delay. 
Corollary 6. Letting $\tau(t)=0, C \neq 0$, and $C^{\tau_{l}}=0$ for $l=$ $1,2, \ldots, m$, if the following inequality holds:

$$
\frac{\ln \eta}{\rho}+\sup \left[\mu\left(I_{N} \otimes M(z)+c(C \otimes A)\right)\right]<0,
$$

where $0<\eta<1$, then the dynamical drive-response network without coupling delays can achieve the projective synchronization.

Corollary 7. Letting $\tau(t)=0, C=0$, and $C^{\tau_{l}} \neq 0$ for $l=$ $2, \ldots, m$, if the following inequality holds:

$$
\frac{\ln \eta}{\rho}+\sup \left[\mu\left(I_{N} \otimes M(z)\right)+\frac{1}{\sqrt{\eta}}\left\|c^{\tau_{1}}\left(C^{\tau_{1}} \otimes A^{\tau_{1}}\right)\right\|\right]<0 ;
$$

where $0<\eta<1$, then the dynamical drive-response network with coupling delays can achieve the projective synchronization.

Remark 8. We consider the equidistant impulsive interval $t_{k}-$ $t_{k-1}=\Delta$ and the impulsive control gain matrix $B_{k}=b I_{n}$, $k=1,2, \ldots$, in Theorem 5 . Then, if the following condition holds: $\Delta<-\left((2|1+b| \ln |1+b|) /\left(|1+b| \sup [\mu(M(z))]+2 \| I_{N} \otimes\right.\right.$ $\left.\left.\gamma \Gamma\left\|+2 \sum_{l=1}^{m}\right\| c^{\tau_{l}}\left(C^{\tau_{l}} \otimes A^{\tau_{l}}\right) \|\right)\right),-2<b<0$, then the projective synchronization is achieved.

Remark 9. The value of scaling factor $\alpha$ has no effect on the error dynamics of the system (see (4)) because the values of $\dot{e}_{i}(t)$ are independent of the scaling factor $\alpha$. So we can arbitrarily direct the scaling factor $\alpha$ onto any desired value.

Remark 10. When $C^{\tau_{l}}=0$ and $\tau=0$ for $l=1,2, \ldots, m$, the drive-response coupled network (1) becomes the driveresponse coupled network discussed in [35-37], and when $C=0, \tau=0$, and $C^{\tau_{l}}=0$ for $l=2, \ldots, m$, the network (1) becomes the network discussed in $[38,39]$. Thus, the obtained results in this paper are more general than those of the above literatures.

Remark 11. Sun et al. [38] studied the projective synchronization in drive-response dynamical networks with timevarying coupling delay, but the time-varying delay in the paper is differential and its derivative is simultaneously required to be not greater than 1 , which is a very strict condition. Obviously, we do not need these limit conditions in theorems and corollaries.

\section{Numerical Simulation}

In this section, numerical simulations are given to verify and demonstrate the effectiveness of the proposed synchronization scheme for synchronizing the drive-response coupled network with time-delayed dynamical nodes and multiple coupling delays onto a scaling factor. We consider the time delay Lorenz chaotic system [32] as the drive system. The Lorenz system with a time delay is described by

$$
\left(\begin{array}{c}
\dot{x} \\
\dot{y} \\
\dot{z}
\end{array}\right)=\left(\begin{array}{c}
\sigma(y-x) \\
(a-z) x-y \\
x y-e z
\end{array}\right)+\gamma\left(\begin{array}{l}
x(t-\tau(t))-x \\
y(t-\tau(t))-y \\
z(t-\tau(t))-z
\end{array}\right),
$$

where $\gamma=10, \tau(t)=5-e^{-t}, \sigma=16, a=40, e=4, \Gamma=I_{2}$. The system (28) with the above parameters is chaotic, as shown in Figure 1.

For simplicity, the drive-response network systems with two terms of time-varying delayed coupling are described as follows:

$$
\begin{aligned}
& \dot{u}^{d}(t)= M(z) \cdot u^{d}(t)+10\left(u^{d}(t-\tau(t))-u^{d}(t)\right) \\
& \dot{z}(t)=x(t) y(t)-z(t)+10(z(t-\tau(t))-z(t)), \dot{u}_{i}^{r}(t)= \\
& M(z) \cdot u_{i}^{r}(t)+10\left(u_{i}^{r}(t-\tau(t))-u_{i}^{r}(t)\right) \\
&+c \sum_{j=1}^{5} c_{i j} A u_{j}^{r}(t) \\
&+\sum_{l=1}^{2} \sum_{j=1}^{5} c^{\tau_{l}} c_{i j}^{\tau_{l}} A^{\tau_{l}} u_{j}^{r}\left(t-\tau_{l}(t)\right), \quad t \neq t_{k}, \\
& \Delta u_{i}^{r}= u_{i}^{r}\left(t_{k}^{+}\right)-u_{i}^{r}\left(t_{k}^{-}\right)=B_{i_{k}}\left[u_{i}^{r}-\alpha u^{d}\right] \\
& t=t_{k}, k=1,2, \ldots,
\end{aligned}
$$

where $M(z)=\left(\begin{array}{cc}-\sigma & \sigma \\ a-z & -1\end{array}\right)$.

The coupling configuration matrices $C=\left(c_{i j}\right)_{N \times N}, C^{\tau_{1}}=$ $\left(c_{i j}^{\tau_{1}}\right)_{N \times N}$, and $C^{\tau_{2}}=\left(c_{i j}^{\tau_{2}}\right)_{N \times N}$ are

$$
\begin{aligned}
C & =\left(\begin{array}{ccccc}
-3 & -1 & 0 & 3 & 1 \\
1 & -3 & 0 & 0 & 2 \\
0 & 1 & 0 & -1 & 0 \\
0 & 1 & 1 & -1 & -1 \\
-2 & 2 & 0 & -1 & 1
\end{array}\right), \\
C^{\tau_{1}} & =\left(\begin{array}{ccccc}
-2 & 0 & 0 & 1 & 1 \\
2 & -4 & 0 & 0 & 2 \\
0 & 2 & 0 & -2 & 0 \\
1 & 1 & 1 & -2 & -1 \\
-2 & 2 & 1 & -2 & 1
\end{array}\right), \\
C^{\tau_{2}} & =\left(\begin{array}{ccccc}
-1 & -1 & 0 & 2 & 0 \\
1 & -3 & 0 & 0 & 2 \\
0 & 1 & 0 & -1 & 0 \\
0 & 1 & 0 & 0 & -1 \\
-2 & 2 & 0 & -1 & 1
\end{array}\right) .
\end{aligned}
$$

According to Theorem 5, we have the result that, for the given scaling factor $\alpha$, if inequalities (6) and (7) can be satisfied, then the error dynamical system (4) will be stabilized at zero equilibrium asymptotically; that is, the projective synchronization of drive-response network systems will be realized.

In the numerical simulations, we assume $c=0.1, c^{\tau_{1}}=$ $0.3, c^{\tau_{2}}=0.2, A=A^{\tau_{l}}=I_{3}, B_{i_{k}}=\operatorname{diag}\{-0.8,-0.8\}$, $(1+b)^{2}=\eta=0.04>0$. The two coupling delays are $\tau_{1}(t)=1+0.2 \sin t$ and $\tau_{2}(t)=0.6+0.1 \sin t$, respectively. After calculations, getting $\lambda_{\text {max }}\left(M^{T}(z)+M(z)\right)=-(\sigma+$ $1)+\sqrt{(\sigma-1)^{2}+(\sigma+a-z)^{2}}<41, \sup [\mu(M(z))]=$ 21.4346,2 $\left\|I_{N} \otimes \gamma \Gamma\right\|=20,2 \sum_{l=1}^{2}\left\|c^{\tau_{l}}\left(C^{\tau_{l}} \otimes A^{\tau_{l}}\right)\right\|=8.3232$, 


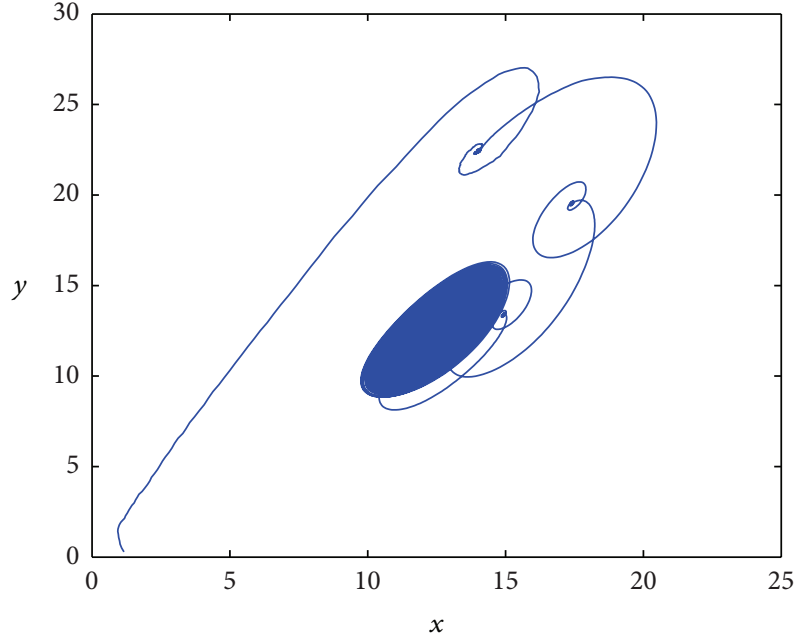

FIGURE 1: Chaotic behavior of the time-delayed system (28).
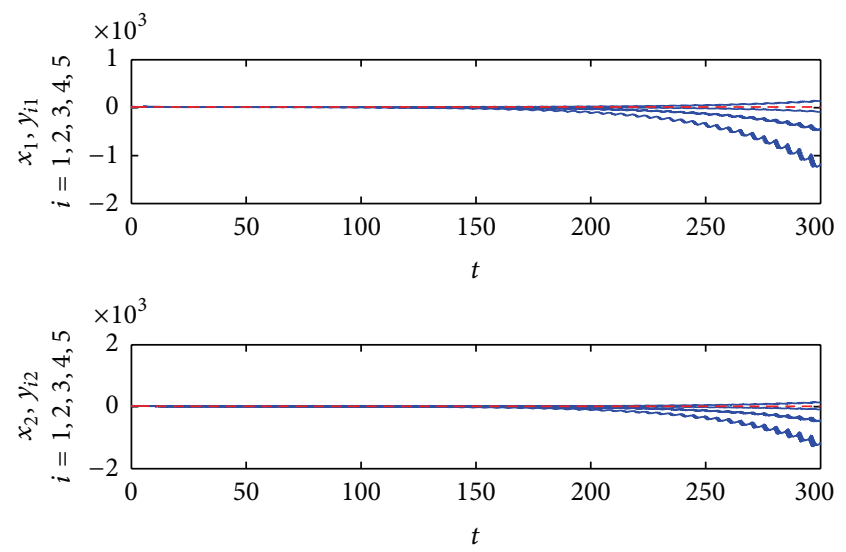

(a)

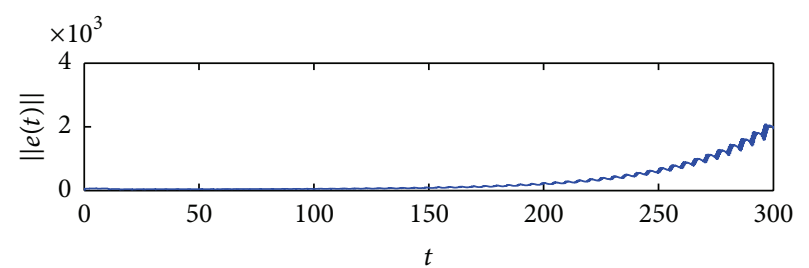

(b)

Figure 2: Evolution of (a) state trajectories of drive and response systems and (b) projective synchronization error without impulsive control when $\tau(t)=5-e^{-t}$.

then, one has $\Delta<0.0197$. Taking the impulsive interval $\Delta=$ $t_{k+1}-t_{k}=0.01$, then, it is easy to verify that all conditions in Remark 8 are satisfied. The projective synchronization error is defined by $\|e(t)\|=\sqrt{\left(x_{i 1}-\alpha x_{1}\right)^{2}+\left(y_{i 2}-\alpha y_{2}\right)^{2}}, i=$ $1,2, \ldots, 5$. When the given scaling factor is $\alpha=2$, Figure 2 shows the evolution process of the error and the states of the drive-response network without impulsive control. From Figure 2, it is easy to see that the projective synchronization

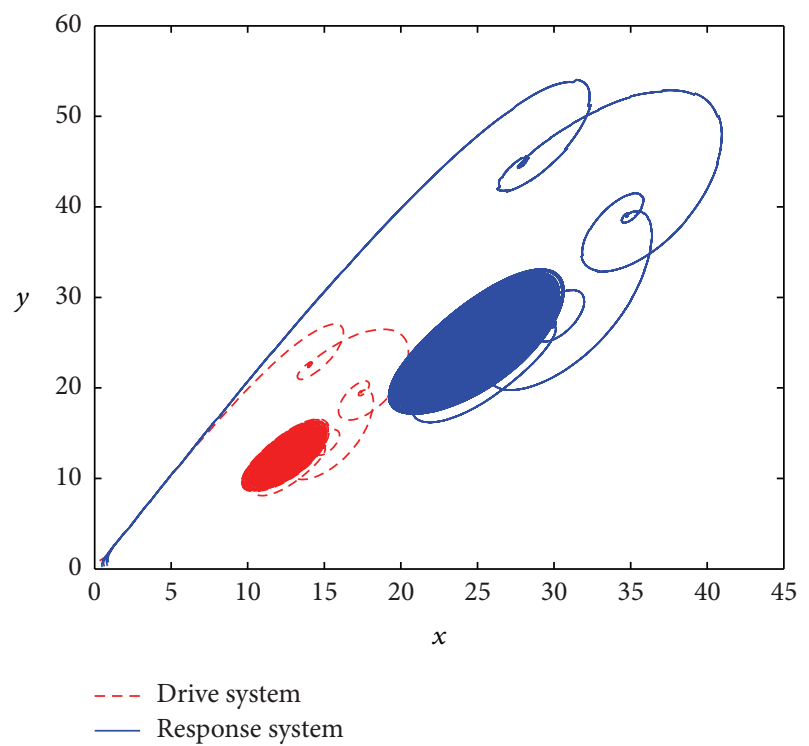

FIGURE 3: The phase plot of $x$ and $y$ plane with $\alpha=2$.
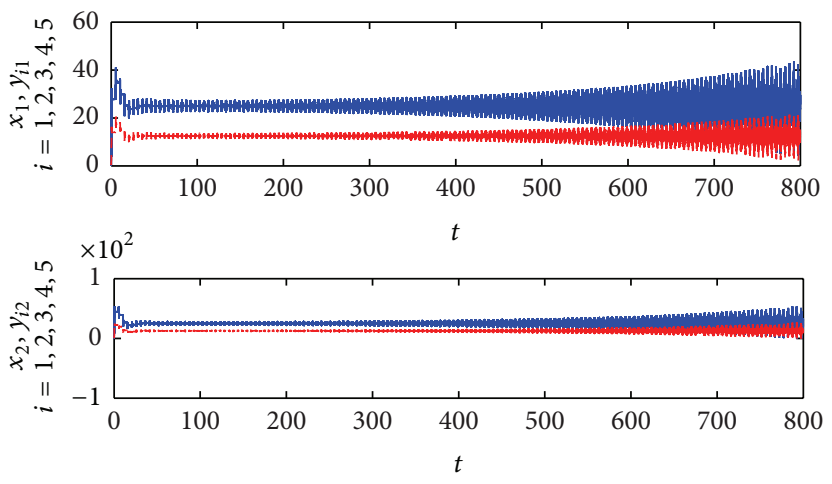

(a)

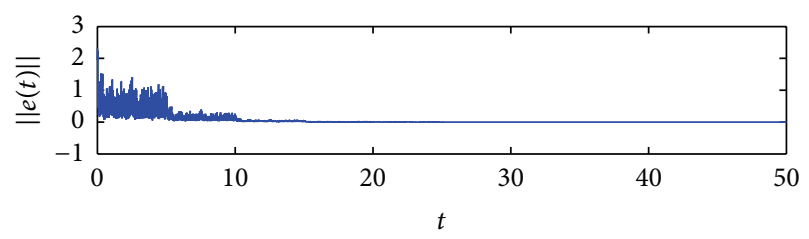

(b)

Figure 4: Evolution of (a) state trajectories of drive (the dash line) and response systems (the solid line) and (b) projective synchronization error under impulsive control.

is not achieved. Figure 3 displays the projective synchronization trajectory of the drive-response dynamical networks. Figure 4 shows the evolution process of the error and the states of the drive-response network with impulsive control. When the given scaling factor is $\alpha=-0.5$, as shown in Figures 5 and 6 , the numerical results show that the impulsive controlling scheme for the drive-response coupled dynamical network with time-varying delays is effective.

Especially, if $\tau=0$, the delayed system (28) becomes the Lorenz system. But if the other conditions are chosen 


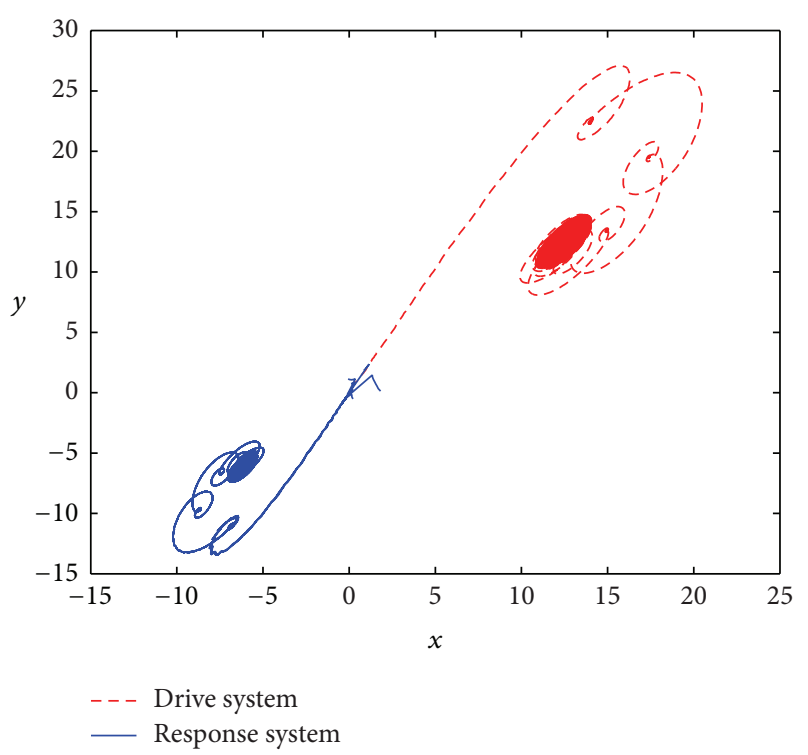

FIGURE 5: The phase plot of $x$ and $y$ plane with $\alpha=-0.5$.
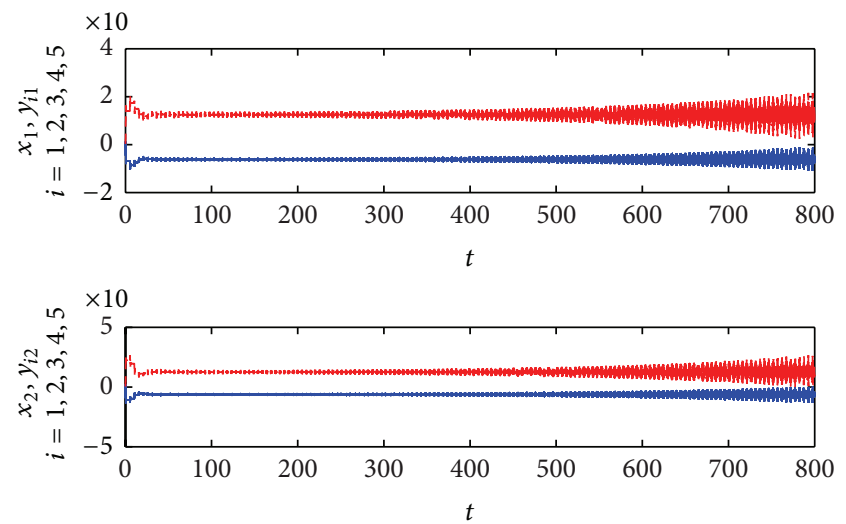

(a)

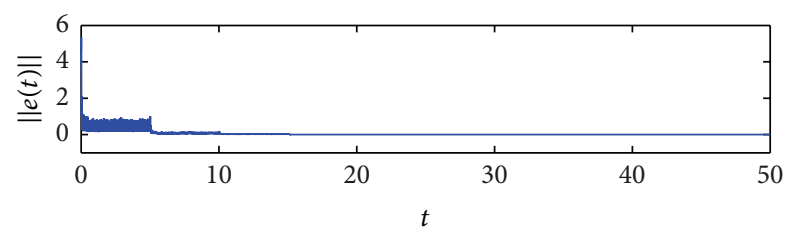

(b)

FIGURE 6: Evolution of (a) state trajectories of drive (the dash line) and response systems (the solid line) and (b) projective synchronization error under impulsive control.

to be the same as above, one has $\Delta<0.0650$. We choose impulsive interval $\Delta=t_{k+1}-t_{k}=0.05$, and the projective synchronization can be obtained with the given scaling factor $\alpha=-2$, and the simulation results are as shown in Figures 7, 8 , and 9. Obviously, the numerical simulations confirm the theoretical analysis.

Remark 12. In this paper, the projective synchronization problem of drive-response coupled dynamical network with
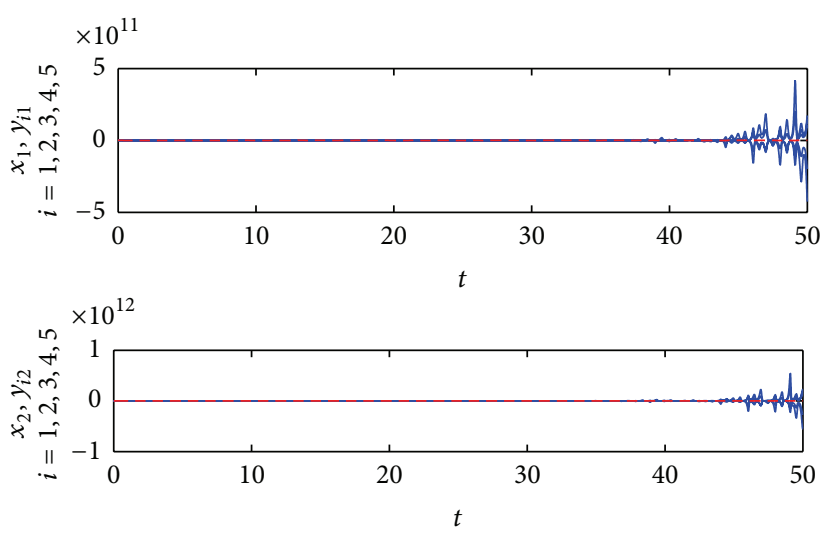

(a)

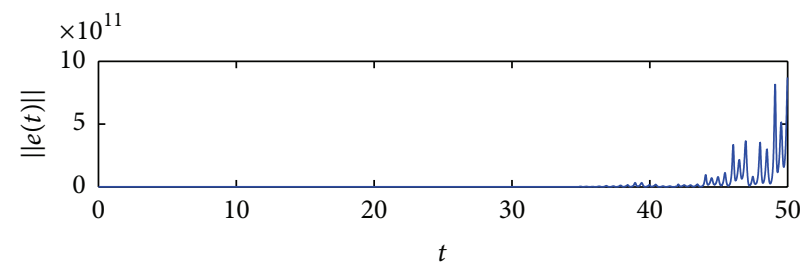

(b)

FIGURE 7: Evolution of (a) state trajectories of drive and response systems and (b) projective synchronization error without impulsive control when $\tau(t)=0$.

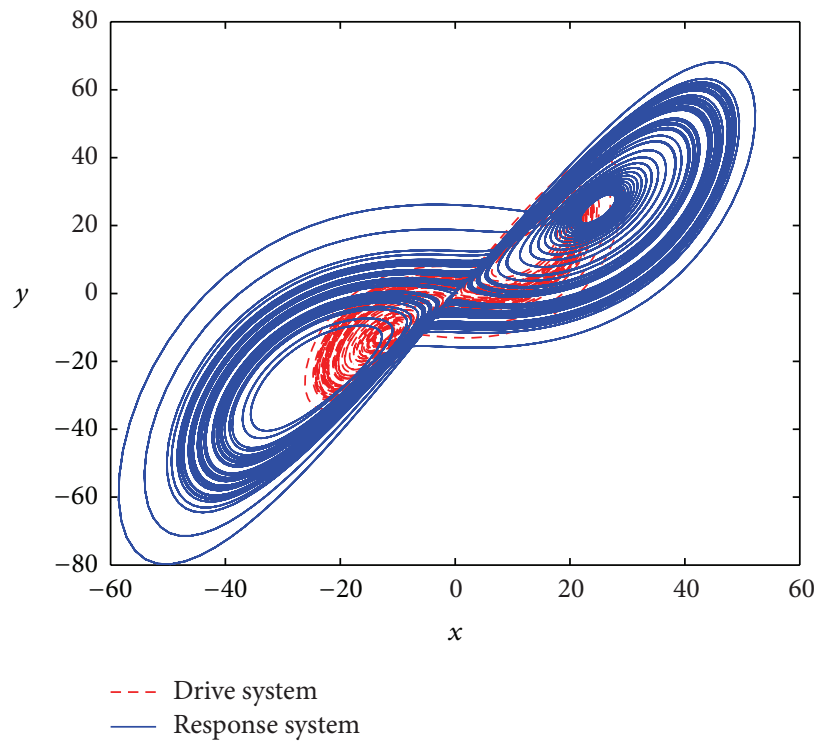

FIGURE 8: The phase plot of $x$ and $y$ plane with $\alpha=-2, \tau(t)=0$.

multiple time-varying delays is studied by employing the impulsive control scheme. As well known, compared with the controller used in adaptive control method [38], the controller used in impulsive method usually is relatively simple and is easy to implement. In the impulsive synchronization, the response networks receive the information from the drive system only in discrete times, which can reduce the information redundancy in the transmitted signal, increase 

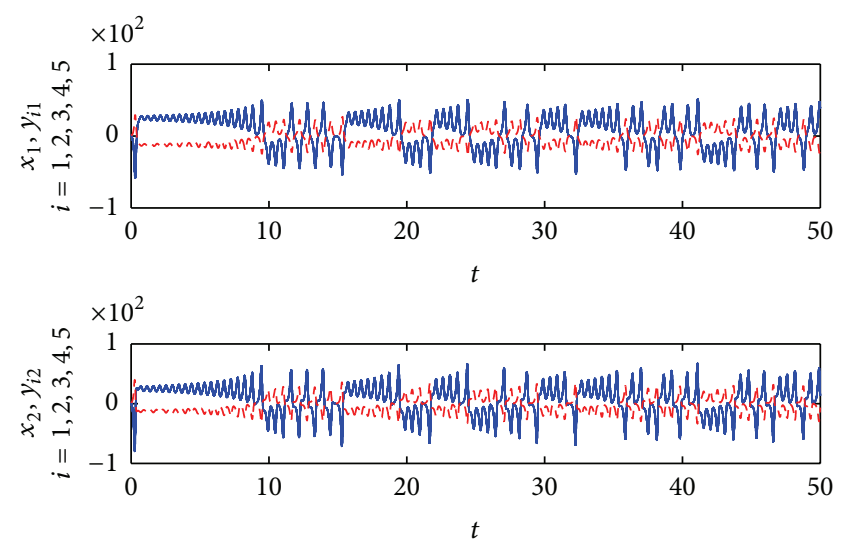

(a)

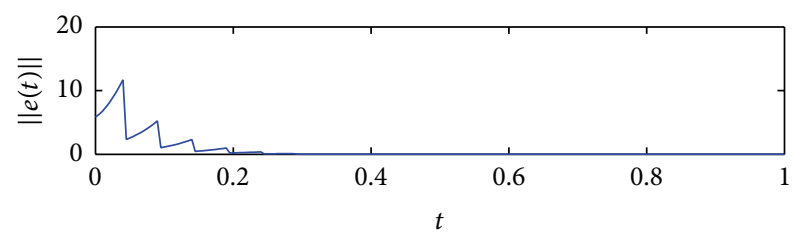

(b)

FIGURE 9: Evolution of (a) state trajectories of drive (the dash line) and response systems (the solid line) and (b) projective synchronization error under impulsive control.

the robustness, and reduce the control cost. Furthermore, from the simulation results, it is clear that the impulsive control scheme is more effective than the adaptive control scheme.

\section{Conclusion}

In this paper, the projective synchronization of driveresponse coupled dynamical network with time delays dynamical nodes and multiple coupling delays has been studied. Some sufficient conditions for realizing the projective synchronization with a scaling factor are established by using the stability analysis of impulsive delayed systems and comparison method. Numerical simulations have also been given to show the effectiveness and the correctness of the theoretical analysis finally.

In the analysis and simulation study in this paper, we fully considered the impact of the time delay element on the projective synchronization of the drive-response network systems. In order to obtain a generic solution of projective synchronization criteria and control scheme, we neglected the particularities of networks. In fact, the dynamic processes of different oscillators are not always unified; as a result their dynamic characteristics under time delay need to be further investigated. Furthermore, we did not consider the environment factors, for example, noise, on the networks, which often affect the synchronization process of the driveresponse network systems. Therefore, with respect to the future work, we will further consider the projective synchronization problem of drive-response network with different dynamics oscillators under different scaling factors. Simultaneously, other environmental factors, for example, the noise, will be taken into account in the study to further improve the robustness of the control solutions.

\section{Conflict of Interests}

The authors declare that they have no conflict of interests.

\section{Acknowledgments}

This work was jointly supported by the National Science Foundation of China (Grant nos. 11102076 and 11202085), the Society Science Foundation from Ministry of Education of China (Grant no. 12YJAZH002), the Natural Science Foundation of Zhejiang Province (Grant no. LY13F030016), and the Foundation of Zhejiang Provincial Education Department (Grant no. Y201328316).

\section{References}

[1] B. A. Huberman and L. A. Adamic, "Growth dynamics of the world-wide web," Nature, vol. 401, no. 6749, pp. 131-132, 1999.

[2] S. H. Strogatz, "Exploring complex networks," Nature, vol. 410, no. 6825 , pp. 268-276, 2001.

[3] W. Sun, "Random walks on generalized Koch networks," Physica Scripta, vol. 88, no. 4, Article ID 045006, 2013.

[4] T. H. Lee, J. H. Park, D. H. Ji, O. M. Kwon, and S. M. Lee, "Guaranteed cost synchronization of a complex dynamical network via dynamic feedback control," Applied Mathematics and Computation, vol. 218, no. 11, pp. 6469-6481, 2012.

[5] F. Sorrentino and E. Ott, "Adaptive synchronization of dynamics on evolving complex networks," Physical Review Letters, vol. 100, no. 11, Article ID 114101, 2008.

[6] T. E. Gorochowski, M. Di Bernardo, and C. S. Grierson, "Evolving enhanced topologies for the synchronization of dynamical complex networks," Physical Review E: Statistical, Nonlinear, and Soft Matter Physics, vol. 81, no. 5, Article ID 056212, 2010.

[7] W. Sun, Y. Yang, C. Li, and Z. Liu, "Synchronization inside complex dynamical networks with double time-delays and nonlinear inner-coupling functions," International Journal of Modern Physics B, vol. 25, no. 11, pp. 1531-1541, 2011.

[8] W. Sun, J. Zhang, and C. Li, "Synchronization analysis of two coupled complex networks with time delays," Discrete Dynamics in Nature and Society, vol. 2011, Article ID 209321, 12 pages, 2011.

[9] B. Shen, Z. Wang, and X. Liu, "Sampled-data synchronization control of dynamical networks with stochastic sampling," IEEE Transactions on Automatic Control, vol. 57, pp. 2644-2650, 2012.

[10] Y. Dong, J. Xian, and D. Han, "New conditions for synchronization in complex networks with multiple time-varying delays," Communications in Nonlinear Science and Numerical Simulation, vol. 18, no. 9, pp. 2581-2588, 2013.

[11] W. Sun and S. Li, "Generalized outer synchronization between two uncertain dynamical networks," Nonlinear Dynamics, 2014.

[12] P. De Lellis, M. Di Bernardo, and F. Garofalo, "Synchronization of complex networks through local adaptive coupling," Chaos, vol. 18, no. 3, Article ID 037110, 2008.

[13] P. DeLellis, M. diBernardo, and F. Garofalo, "Novel decentralized adaptive strategies for the synchronization of complex networks," Automatica, vol. 45, no. 5, pp. 1312-1318, 2009. 
[14] M. M. Asheghan, J. Míguez, M. T. Hamidi-Beheshti, and M. S. Tavazoei, "Robust outer synchronization between two complex networks with fractional order dynamics," Chaos, vol. 21, no. 3, Article ID 033121, 2011.

[15] B. Wang, J. Jiang, and H. Yu, "Adaptive synchronization of fractional-order memristor-based Chua's system," Science and Control Engineering, vol. 2, pp. 291-296, 2014.

[16] Z.-H. Guan, D. J. Hill, and J. Yao, "A hybrid impulsive and switching control strategy for synchronization of nonlinear systems and application to Chua's chaotic circuit," International Journal of Bifurcation and Chaos, vol. 16, no. 1, pp. 229-238, 2006.

[17] P. Li, J. Cao, and Z. Wang, "Robust impulsive synchronization of coupled delayed neural networks with uncertainties," Physica A: Statistical Mechanics and its Applications, vol. 373, pp. 261-272, 2007.

[18] J. Zhou, Q. Wu, and L. Xiang, "Impulsive pinning complex dynamical networks and applications to firing neuronal synchronization," Nonlinear Dynamics, vol. 69, pp. 1393-1403, 2012.

[19] Q. Zhang and J. Zhao, "Projective and lag synchronization between general complex networks via impulsive control," Nonlinear Dynamics, vol. 67, no. 4, pp. 2519-2525, 2012.

[20] Y. Fang, K. Yan, and K. Li, "Impulsive synchronization of a class of chaotic systems," Systems Science and Control Engineering, vol. 2, no. 1, pp. 55-60, 2014.

[21] T. Chen, X. Liu, and W. Lu, "Pinning complex networks by a single controller," IEEE Transactions on Circuits and Systems I: Regular Papers, vol. 54, no. 6, pp. 1317-1326, 2007.

[22] J. Zhou, J.-A. Lu, and J. Lü, "Pinning adaptive synchronization of a general complex dynamical network," Automatica, vol. 44, no. 4, pp. 996-1003, 2008.

[23] S. Wang, C. He, and H. Yao, "Cluster anti-synchronization of complex networks with nonidentical dynamical nodes," Journal of Applied Mathematics, vol. 2012, Article ID 347570, 12 pages, 2012.

[24] S. Wang, H. Yao, and M. Sun, "Cluster synchronization of timevarying delays coupled complex networks with nonidentical dynamical nodes," Journal of Applied Mathematics, vol. 2012, Article ID 958405, 12 pages, 2012.

[25] S. Wang and H. Yao, "The effect of control strength on lag synchronization of nonlinear coupled complex networks," Abstract and Applied Analysis, vol. 2012, Article ID 810364, 11 pages, 2012.

[26] Q. Hu, H. Peng, Y. Wang, Z. Hu, and Y. Yang, "Pinning adaptive synchronization of complex dynamical network with multilinks," Nonlinear Dynamics, vol. 69, pp. 1813-1824, 2012.

[27] S. Cai, Z. Liu, F. Xu, and J. Shen, "Periodically intermittent controlling complex dynamical networks with time-varying delays to a desired orbit," Physics Letters A: General, Atomic and Solid State Physics, vol. 373, no. 42, pp. 3846-3854, 2009.

[28] R. Mainieri and J. Rehacek, "Projective synchronization in three-dimensional chaotic systems," Physical Review Letters, vol. 82, no. 15, pp. 3042-3045, 1999.

[29] D. Xu, "Control of projective synchronization in chaotic systems," Physical Review E, vol. 63, pp. 27201-27204, 2001.

[30] N. Vasegh and F. Khellat, "Projective synchronization of chaotic time-delayed systems via sliding mode controller," Chaos, Solitons and Fractals, vol. 42, no. 2, pp. 1054-1061, 2009.

[31] Z.-L. Wang, "Projective synchronization of hyperchaotic Lü system and Liu system," Nonlinear Dynamics, vol. 59, no. 3, pp. 455-462, 2010.
[32] J. Cao, D. W. C. Ho, and Y. Yang, "Projective synchronization of a class of delayed chaotic systems via impulsive control," Physics Letters A: General, Atomic and Solid State Physics, vol. 373, no. 35, pp. 3128-3133, 2009.

[33] S. Zheng, Q. Bi, and G. Cai, "Adaptive projective synchronization in complex networks with time-varying coupling delay," Physics Letters A: General, Atomic and Solid State Physics, vol. 373, no. 17, pp. 1553-1559, 2009.

[34] S. Zheng, "Analyzing projective synchronization on different scaling factors in a drive-response coupling dynamical network with time-varying delays," Nonlinear Dynamics, vol. 70, no. 1, pp. 709-719, 2012.

[35] M. Hu, Y. Yang, Z. Xu, R. Zhang, and L. Guo, "Projective synchronization in drive-response dynamical networks," Physica A: Statistical Mechanics and its Applications, vol. 381, no. 1-2, pp. 457-466, 2007.

[36] M. Hu, Z. Xu, and Y. Yang, "Projective cluster synchronization in drive-response dynamical networks," Physica A: Statistical Mechanics and its Applications, vol. 387, no. 14, pp. 3759-3768, 2008.

[37] Y. Zhao and Y. Yang, "The impulsive control synchronization of the drive-response complex system," Physics Letters A: General, Atomic and Solid State Physics, vol. 372, no. 48, pp. 7165-7171, 2008.

[38] M. Sun, C. Zeng, and L. Tian, "Projective synchronization in drive-response dynamical networks of partially linear systems with time-varying coupling delay," Physics Letters A: General, Atomic and Solid State Physics, vol. 372, no. 46, pp. 6904-6908, 2008.

[39] J. Chen, L. Jiao, J. Wu, and X. Wang, "Projective synchronization with different scale factors in a drivenresponse complex network and its application in image encryption," Nonlinear Analysis: Real World Applications, vol. 11, no. 4, pp. 3045-3058, 2010.

[40] S. Zheng, "Adaptive-impulsive projective synchronization of drive-response delayed complex dynamical networks with time-varying coupling," Nonlinear Dynamics, vol. 67, no. 4, pp. 2621-2630, 2012.

[41] Z. Yang and D. Xu, "Stability analysis and design of impulsive control systems with time delay," IEEE Transactions on Automatic Control, vol. 52, no. 8, pp. 1448-1454, 2007. 


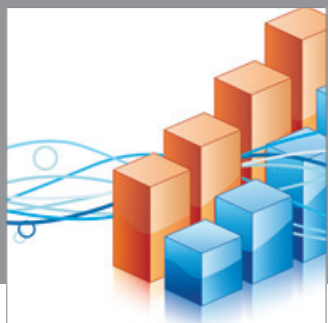

Advances in

Operations Research

mansans

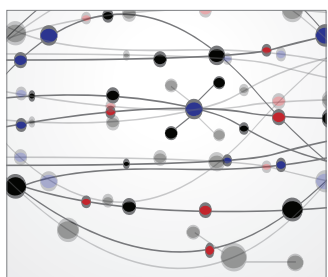

The Scientific World Journal
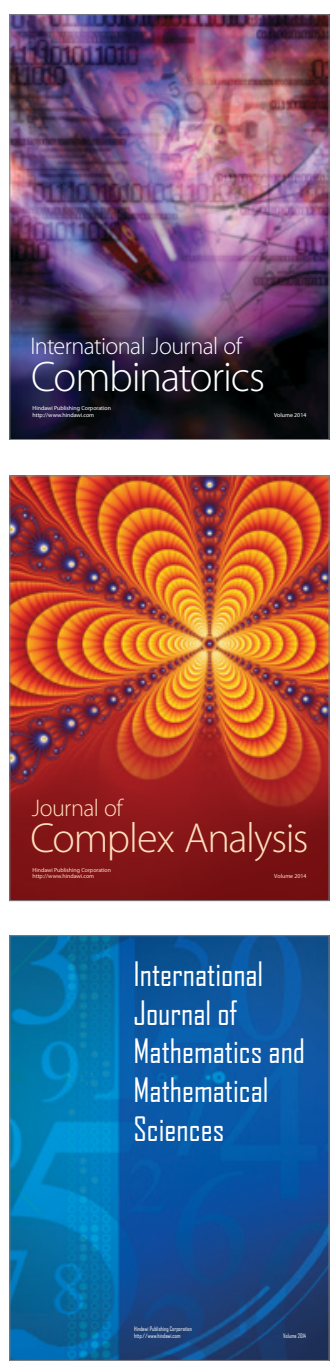
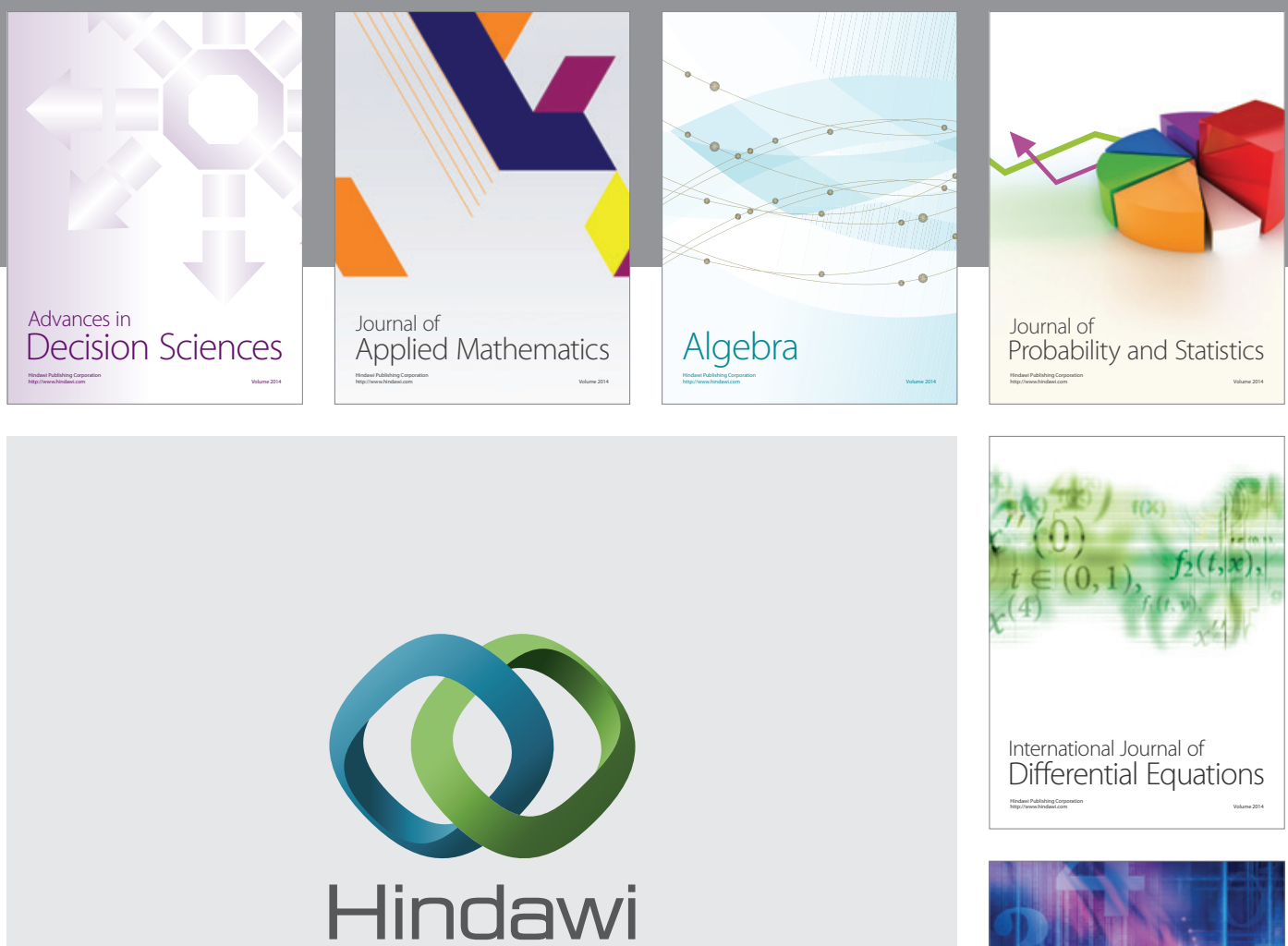

Submit your manuscripts at http://www.hindawi.com
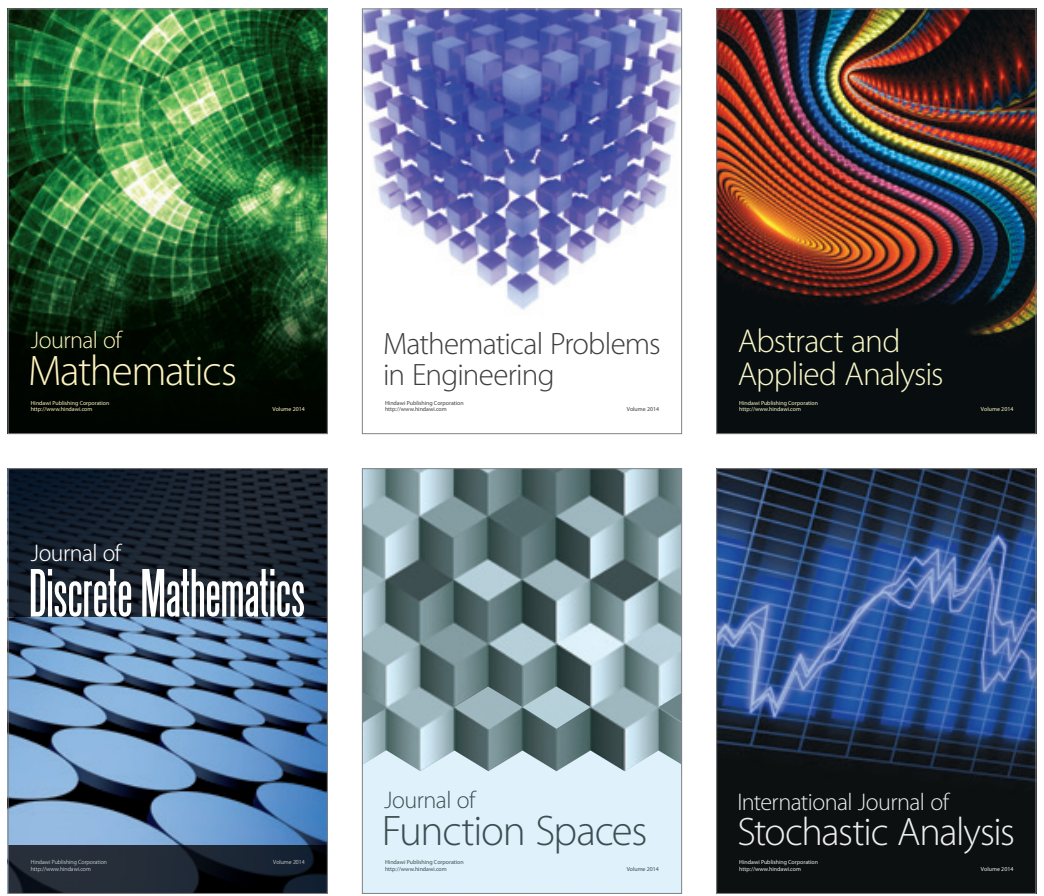

Journal of

Function Spaces

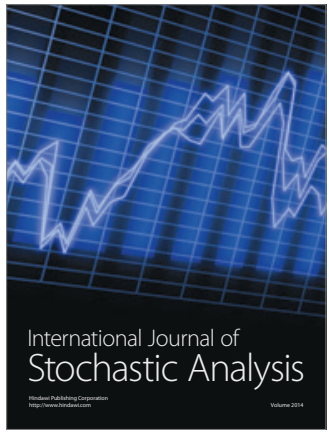

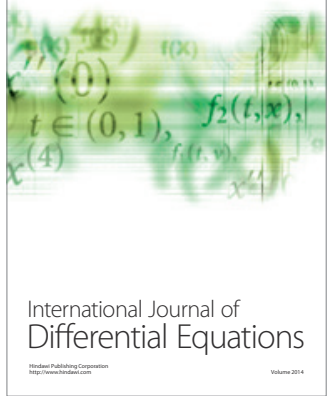
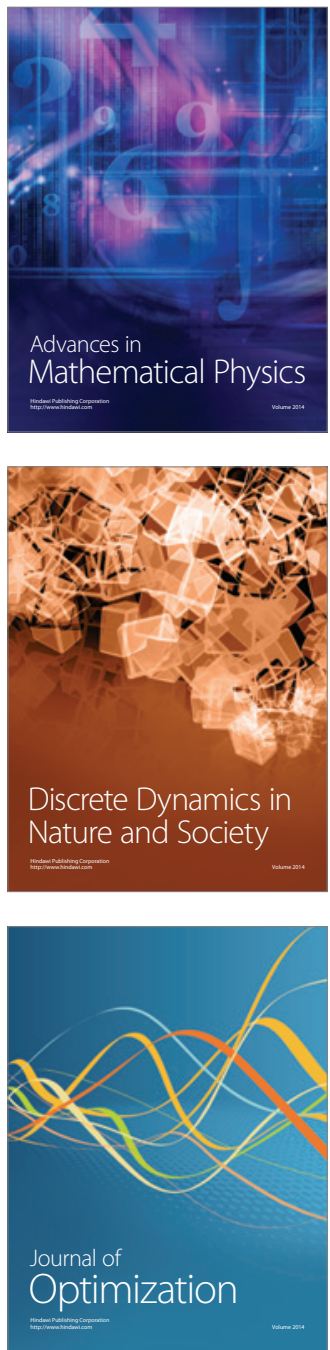